\title{
Directionally dependent horizontal-to-vertical spectral ratios of microtremors at Onahama, Fukushima, Japan
}

\author{
Shinichi Matsushima ${ }^{1 *} \mathbb{D}$, Hiroyuki Kosaka ${ }^{2}$ and Hiroshi Kawase ${ }^{1}$
}

\begin{abstract}
As observational evidence of 3-D microtremor horizontal-to-vertical spectral ratios (MHVRs), previous studies have shown that a significant directional dependency is observed in and around Uji campus, Kyoto University, Japan. This directional dependence is considered to be the result of 2-D basin structure. In this study, we observed microtremors around a strong motion observation site of the Port and Harbor Research Institute in Onahama, Japan, and found that directional dependence of MHVRs exists in some parts of the area around the site. The directional dependence is more apparent and has a higher dominant frequency, at around $5 \mathrm{~Hz}$, relative to those observed in $\mathrm{Uji}$, at around $0.5 \mathrm{~Hz}$. We defined a parameter $\gamma$, which we refer to as the "directionally dependent coefficient" to indicate the magnitude of difference between the two orthogonal components which implies the directional dependence of the MHVRs. We rotated the axes and calculated $\gamma$ for each angle and searched for the orientation that gave the largest $\gamma$ at a point. Points for which the axis with larger MHVR amplitude among the two axes is oriented in the NS direction are aligned in the NS direction, while points for which the axis with larger MHVR amplitude is oriented in the EW direction are aligned in the EW direction. The distribution of points with large $\gamma$ formed a T-shaped distribution. We calculated the analytical and numerical MHVRs in order to simulate the observed MHVRs and succeeded in showing the existence of a narrow wedge. From these results, we conclude that a wedge-like lateral heterogeneity exists in the shallow subsurface of the studied area, parallel to the direction of the axis of the larger MHVR amplitude.
\end{abstract}

Keywords: Horizontal-to-vertical spectral ratio, Directional dependency, Microtremor, Lateral heterogeneity, Onahama

\section{Introduction}

As observational evidence of 3-D microtremor horizontal-to-vertical spectral ratios (MHVRs), previous studies (e.g., Matsushima et al. 2014) have shown that a significant directional dependency is observed in and around Uji campus, Kyoto University, Japan, where the bedrock depth varies from east to west by roughly $400 \mathrm{~m}$ over a distance of $1 \mathrm{~km}$. The microtremors recorded at Uji campus showed that if we compare the MHVRs for the two orthogonal components, i.e., NS/UD and EW/UD, the NS/UD has higher peak amplitude than that of EW/UD,

\footnotetext{
*Correspondence: matsushima@sds.dpri.kyoto-u.ac.jp

${ }^{1}$ Disaster Prevention Research Institute, Kyoto University, Gokasho, Uji,

Kyoto 611-0011, Japan

Full list of author information is available at the end of the article
}

and EW/UD has higher peak frequency than that of NS/ UD. This directional dependence is considered to be the result of 2-D basin structure (Matsushima et al. 2014). In this study, we observed microtremors around a strong motion observation site of the Port and Harbor Research Institute strong motion network (e.g., Nozu and Wakai 2011) in Onahama, Fukushima, Japan. This is one of the sites that were selected for detailed study for the PRENOLIN (improvement of prediction of soil nonlinear effects caused by strong seismic motion) Project (Régnier et al. 2015). In order to investigate the underground structure of the site, we conducted microtremor observation for estimating the phase velocity by spatial autocorrelation method (Aki 1957) or estimating the velocity structure from MHVRs, and multi-channel analysis of surface waves (MASW) in and around the premises of 
the Onahama Port Office of the Ministry of Land, Infrastructure, Transport and Tourism (MLIT), where the strong motion observation site is located. We detected directional dependence in some parts of the area around the site, so we conducted dense microtremor observations to detect the distribution of the directionally dependent MHVRs with the aim of finding the cause of the directionality. The observed MHVRs showed a significant directional dependency and has a higher dominant frequency, at around $5 \mathrm{~Hz}$, compared to those observed in Uji, at around $0.5 \mathrm{~Hz}$. The results from MASW also showed some heterogeneity in the shallow subsurface structure. In order to clarify the effect of the heterogeneous subsurface structure to the MHVRs, we defined a parameter that is capable of indicating the magnitude of directional dependence from the observed MHVRs.

\section{Observed MHVRs}

Location of target site and microtremor observation points The target site of this study is the Onahama strong motion observation site of Port and Harbor Research Institute strong motion network. This observation site is located in the premises of the Onahama Port Office of MLIT. Figure 1 shows the location of Onahama, Fukushima, Japan, and the Onahama Port Office by orange circles. The red rectangle in Fig. 1 denotes the target area of this study. Inside the red rectangle, we first made microtremor measurements within the premises of the Onahama Port Office, as shown in Fig. 2. By analyzing the MHVRs obtained at the observation points in Fig. 2, we found a possibility of the existence of very local lateral heterogeneity in some part of the observed area, but the boundary of the heterogeneity was not clear. So we additionally observed microtremor at points outside of the premises of the Onahama Port Office. Figure 3 shows the distribution of the observed microtremor points where the gray hatched block surrounded by thicker lines denotes the premises of the Onahama Port Office shown in Fig. 2.

\section{Outline of microtremor observation}

The microtremor observations at each point shown in Figs. 2 and 3 were conducted using a portable accelerometer with amplifier SMAR-6A3P manufactured by Akashi Corp. (now Mitutoyo Corp.) combined with data logger LS-8800 manufactured by Hakusan Corp. The time sampling and amplification were set to $200 \mathrm{~Hz}$ and 500 times, respectively. Each instrument was oriented to magnetic north according to the compass. For each line, we made simultaneous recording along the line by using the necessary number of instruments. The clock of each instrument was calibrated using the GPS signal in order to obtain an accurate time. We observed for $30 \mathrm{~min}$ for each line.

\section{MHVRs obtained from microtremor observations}

In order to determine the directional dependence, we calculate the MHVRs separately for the two horizontal components, namely NS/UD and EW/UD. First we calculate the Fourier spectrum of each component and smooth the spectrum with a Parzen window with a bandwidth of $0.1 \mathrm{~Hz}$. Then we take the ratio of each horizontal component to the UD component. The MHVRs for the two components at the sites within the premises of

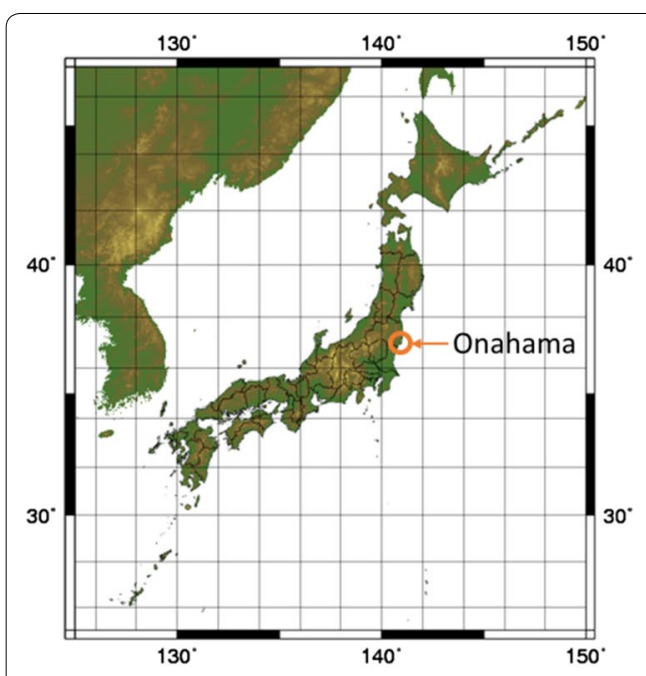

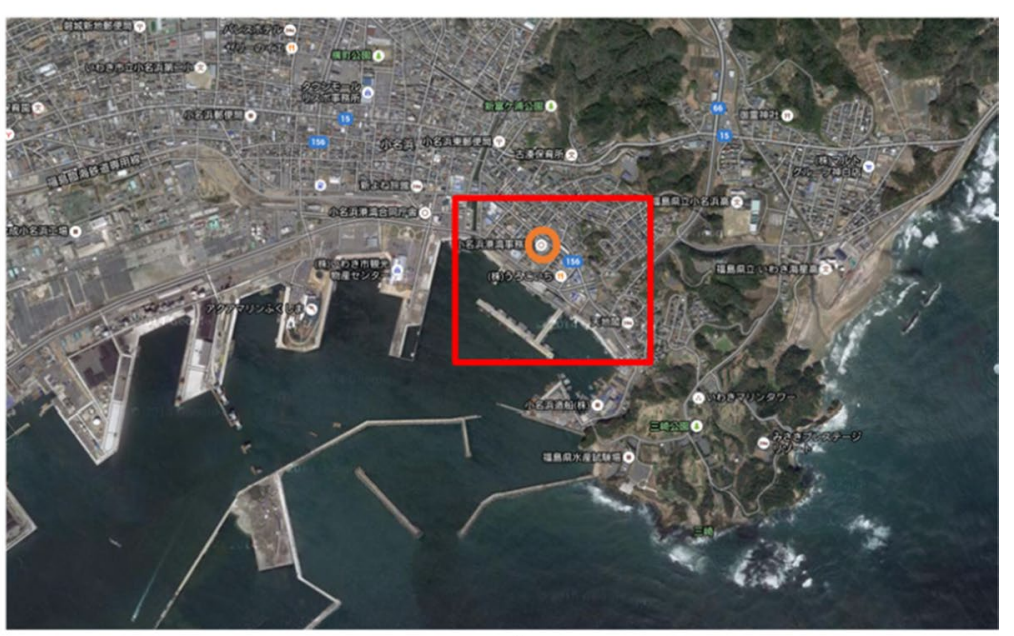

b

Fig. 1 Location of the target site of this study, a Onahama, Fukushima, Japan, and $\mathbf{b}$ Onahama Port Office of MLIT, denoted by orange circles. The red rectangle denotes the target area of this study 


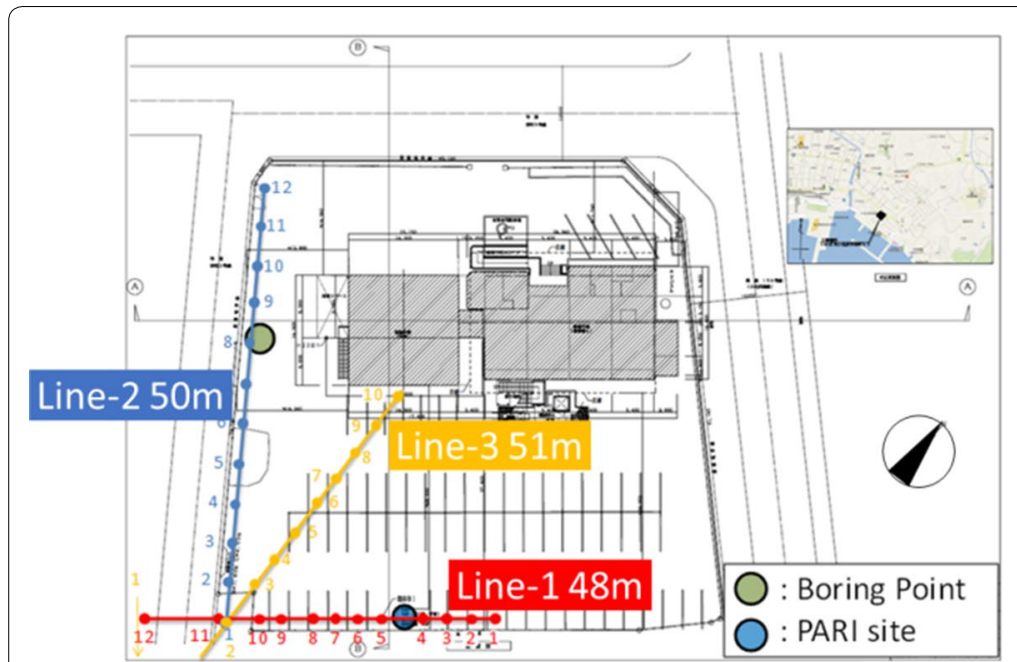

a

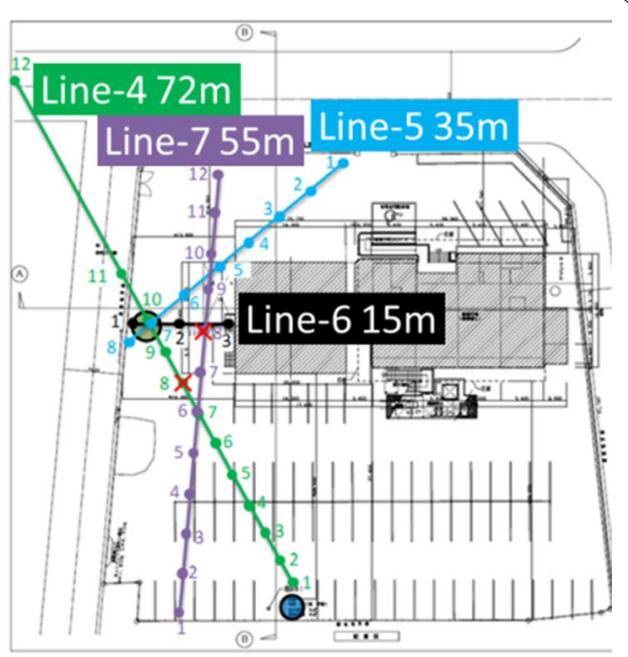

b

Fig. 2 The microtremor observation points within the premises of the Onahama Port Office for $\mathbf{a}$ Line-1 to Line-3 and $\mathbf{b}$ Line-4 to Line-7. The blue and green circles denote the strong motion observation site and the boring point by the PRENOLIN Project (Régnier et al. 2015), respectively

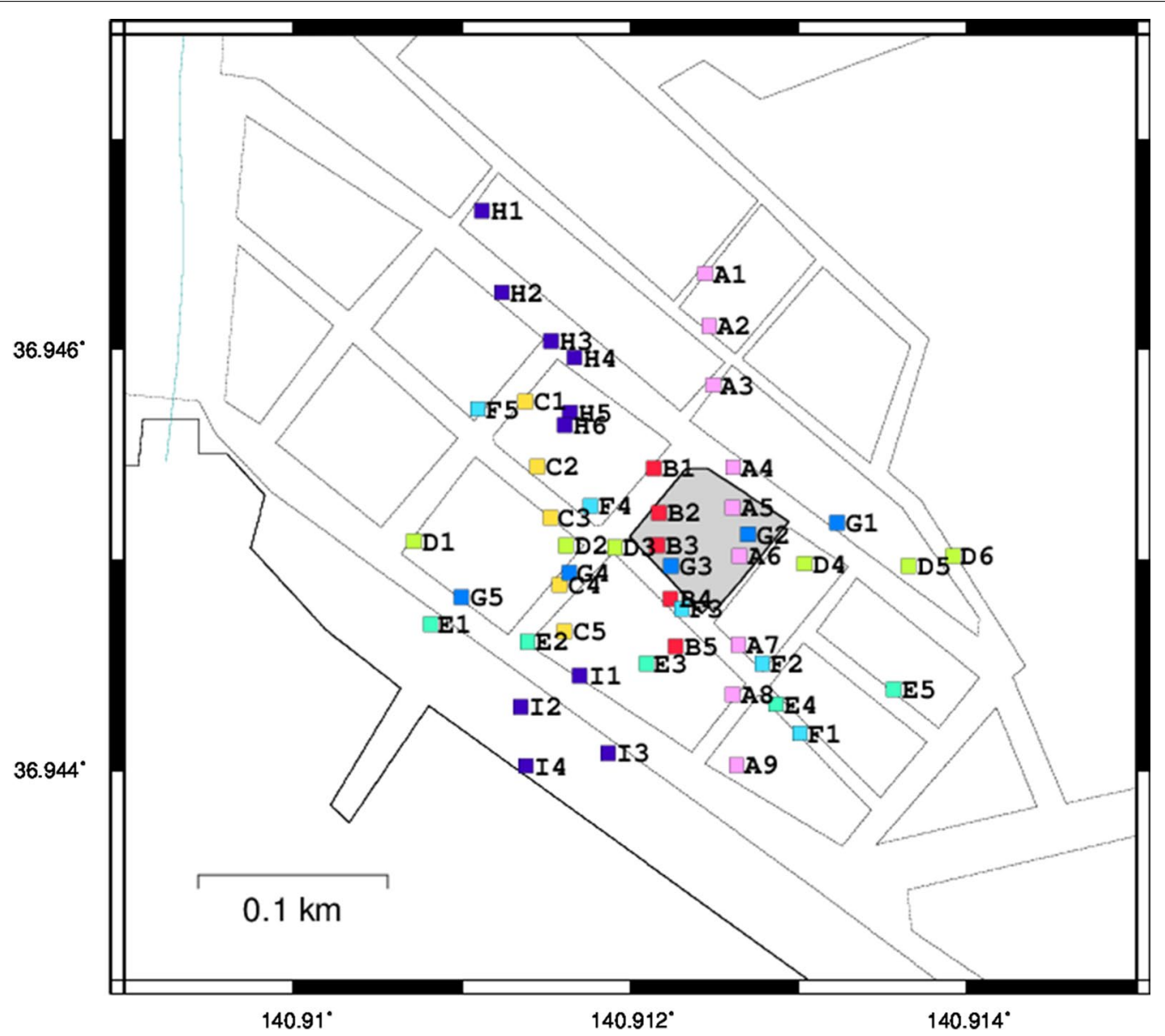

Fig. 3 The microtremor observation points outside of the premises of the Onahama Port Office for Line-A to Line-l. The dotted lines are local roads picked originally from general maps. The gray hatched block surrounded by thicker lines denotes the premises of the Onahama Port Office shown in Fig. 2 
the Onahama Port Office are shown in Figs. 4 and 5 for Line- 3 and Line- 4 , respectively. In these and subsequent figures, the NS/UD and EW/UD are indicated in blue and red lines, respectively. For Line-3, which is aligned in the NS direction, the difference between the two components is nominal for points 3-1 to $3-4$, but from points 3-7 to 3-9 the difference is significant at the peak frequency of about $5 \mathrm{~Hz}$. If we look in detail, we can notice that for the southern points 3-1 to 3-3, there is one dominant peak at frequency slightly over $10 \mathrm{~Hz}$ for both components, but as we move to the north the peak frequency changes. At point 3-4, an additional peak at $8 \mathrm{~Hz}$ appears and then at point 3-5 the peak at $10 \mathrm{~Hz}$ diminishes and only the peak at $8 \mathrm{~Hz}$ remains. Then at point $3-6$, the peak at $8 \mathrm{~Hz}$ diminishes and a small peak at about $5 \mathrm{~Hz}$ emerges. After this transition zone, a clear sharp peak at about $5 \mathrm{~Hz}$ for
NS/UD can be seen at points 3-7 to 3-10, but hardly any peak can be identified for EW/UD at these points. As for Line-4 in Fig. 5, which is aligned in the EW direction, we see a sharp peak at $5 \mathrm{~Hz}$ in NS/UD for points 4-1 to 4-10. At point 4-11, this peak becomes smaller and at point 4-12 the peak of NS/UD becomes close to the peak of EW/UD in amplitude. This may be an indication that some lateral heterogeneity exists in the subsurface structure crossing Line-3 and extending parallel to Line4. To investigate the extent of the assumed irregularity, we conducted microtremor measurements around the premises of the Onahama Port Office at the points shown in Fig. 3. For Line-A shown in Fig. 6, which is aligned in the NS direction and goes through the premises, we can see the same characteristics at points A5 and A6, which are located inside the premises, as those of points 3-7 and

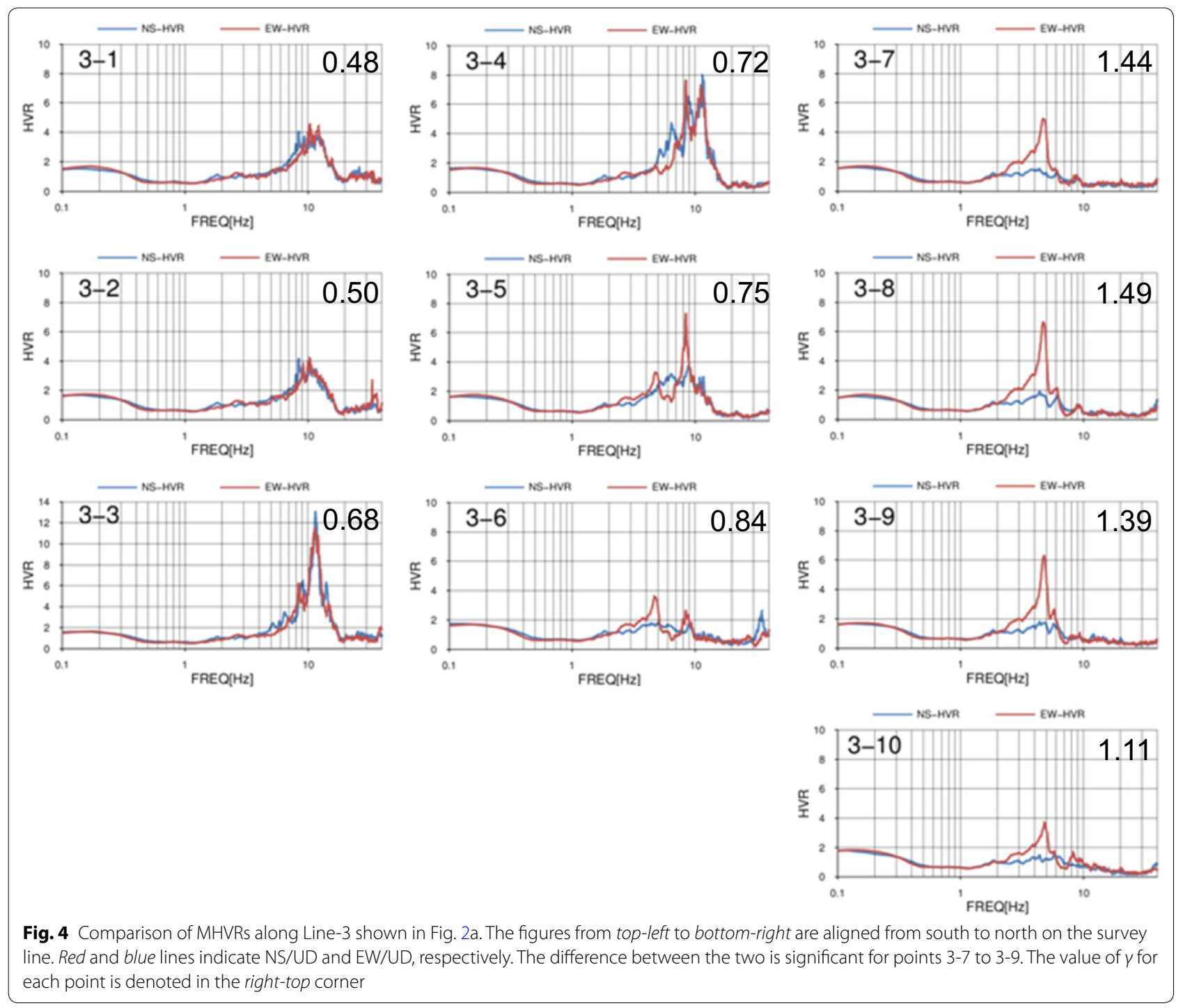




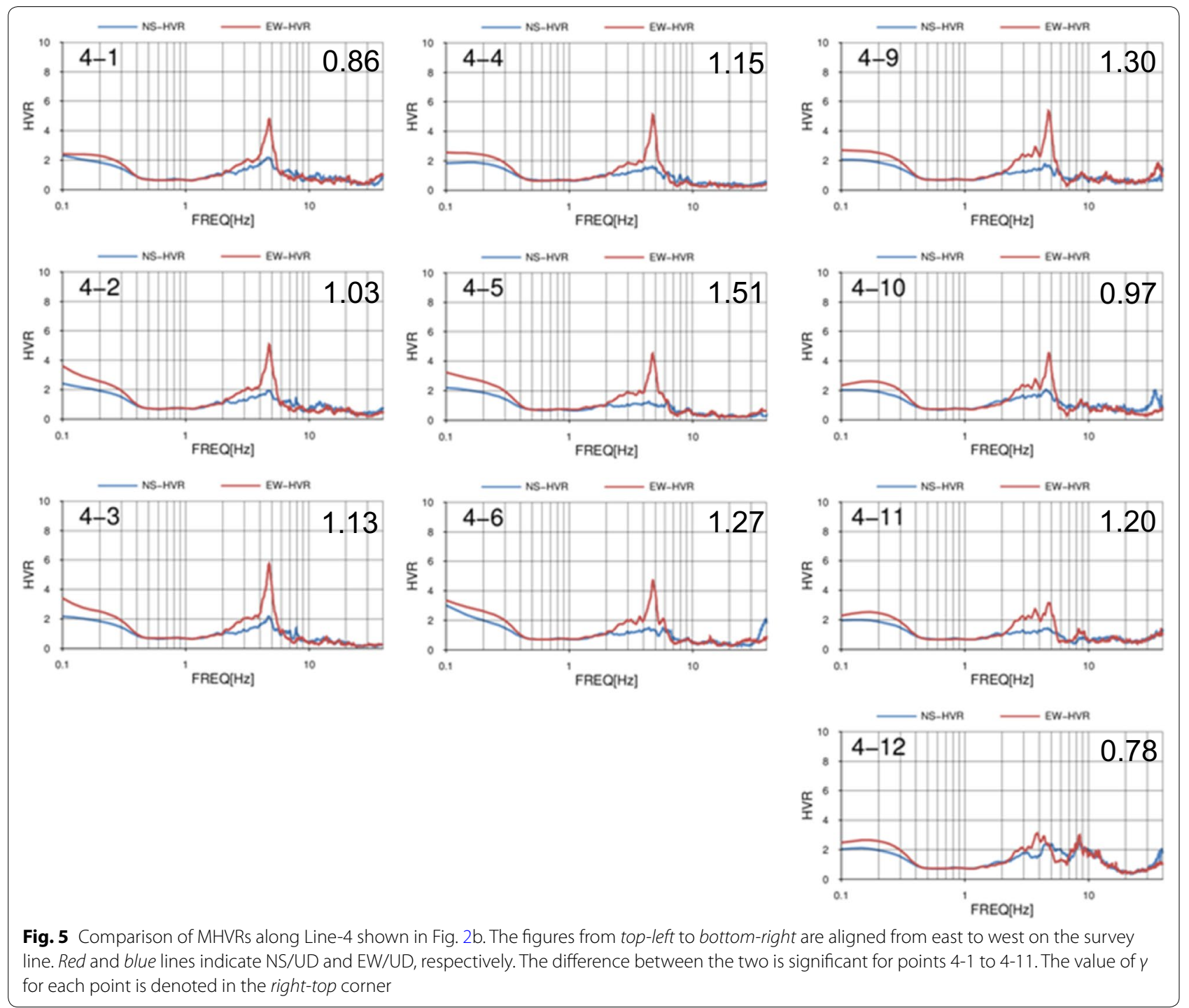

4-6, but for points outside of the premises, e.g., A1 to A4 and A7 to A9, there is no significant difference between NS/UD and EW/UD.

In contrast, for Line- $\mathrm{C}$ shown in Fig. 7 , which is also aligned in the NS direction but off to the west from the premises, we can also see a significant directional dependence but the components are opposite compared to those of Figs. 4, 5 and 6. Only the EW/UD has a clear peak at around $3.5 \mathrm{~Hz}$, which is lower than the peak frequencies for NS/UD of Figs. 4, 5 and 6.

\section{Directionally dependent MHVRs}

Distribution of peak frequencies and peak amplitudes

In order to understand the distribution of the MHVRs that show directional dependence, we plot the peak frequency and the peak amplitude for each site in Fig. 8. The colors indicate the peak frequency, and the size of the circles indicates the amplitude at the peak frequency. For sites with peak frequencies $3-5 \mathrm{~Hz}$ shown in red and orange colors, it can be noticed that size of the red or orange circles is different between NS/UD and EW/UD. For points where peak frequency is around $5 \mathrm{~Hz}$ (orange circles), the peak amplitude of EW/UD is larger than those of NS/UD, and it seems they are aligned in a line from east to west. On the other hand, for points where peak frequency is around $3 \mathrm{~Hz}$ (red circles), the peak amplitude of NS/UD is larger than those of EW/UD, and it seems they are aligned in a line from north to south with limited extent for both directions.

\section{Directionally dependent coefficient $\gamma$}

As we saw in the previous section, the peak amplitudes of NS/UD and EW/UD are significantly different at some sites and quite similar at other sites. We define a 

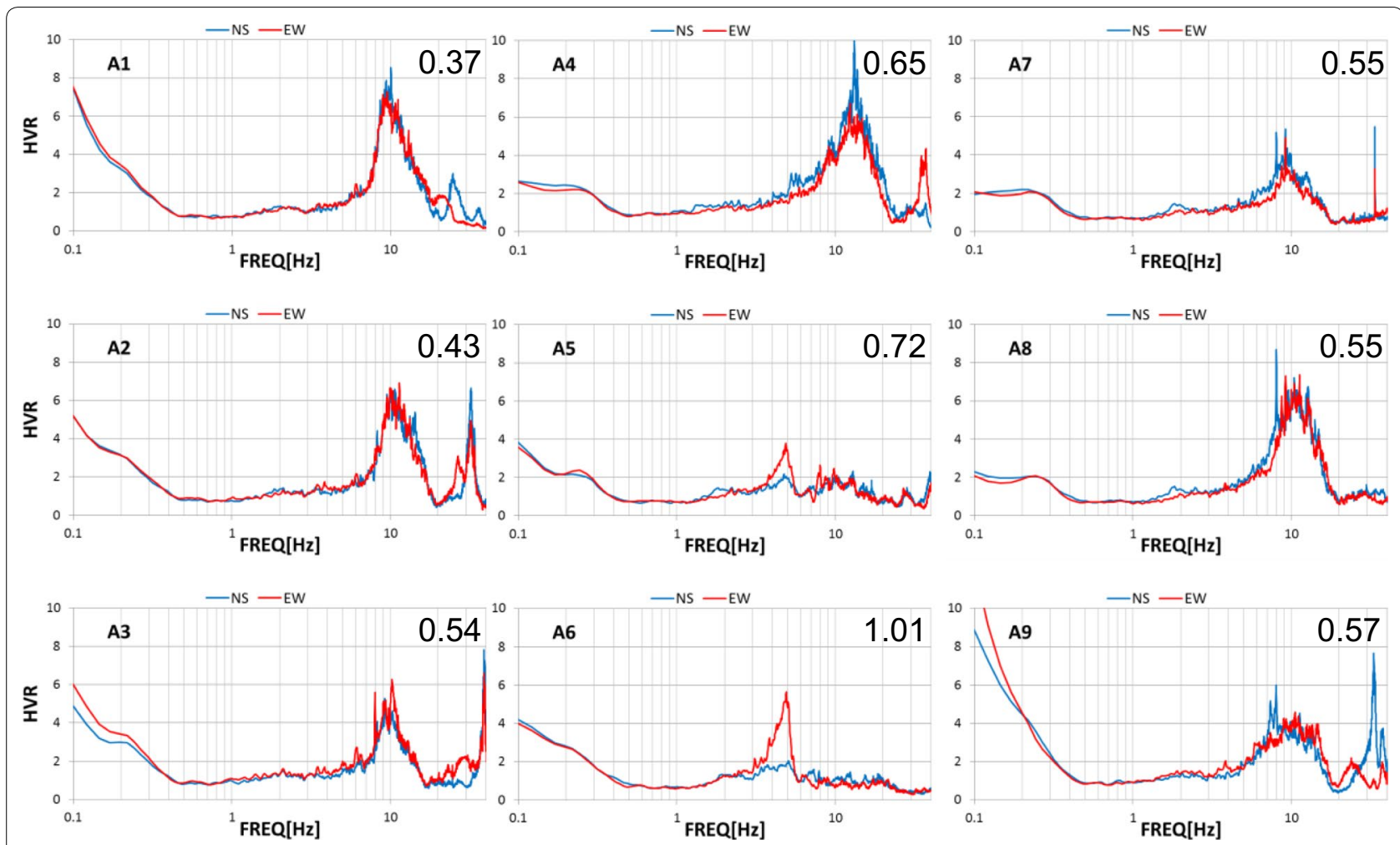

Fig. 6 Comparison of MHVRs along Line-A shown in Fig. 3. The figures from top-left to bottom-right are aligned from north to south on the survey line. Red and blue lines indicate NS/UD and EW/UD, respectively. The difference between the two is significant for points A-5 and A-6. The value of $y$ for each point is denoted in the right-top corner

parameter $\gamma$, which we refer to as "directionally dependent coefficient" to classify the sites according to their directional dependence. This coefficient $\gamma$ is defined by Eq. 1,

$$
\gamma=\frac{1}{N} \sum_{f_{1}}^{f_{2}} \sqrt{\frac{\operatorname{abs}\left\{(\mathrm{NS}(f) / \mathrm{UD}(f))^{2}-(\operatorname{EW}(f) / \mathrm{UD}(f))^{2}\right\}}{\{\min (\mathrm{NS}(f) / \mathrm{UD}(f), \operatorname{EW}(f) / \mathrm{UD}(f))\}^{2}}}
$$

where $\mathrm{NS}(f) / \mathrm{UD}(f)$ and $\operatorname{EW}(f) / \mathrm{UD}(f)$ are the spectral ratios at each frequency $f, f_{1}$ and $f_{2}$ are the low and high frequency limit of the frequency range of interest, and $N$ is the number of data within the range. In this study, we use $f_{1}=1.0 \mathrm{~Hz}$ and $f_{2}=6.0 \mathrm{~Hz}$. Figure 9 shows a comparison of the coefficient $\gamma$ for lines Line-A to Line-I and Line-3 to Line-4. A larger value of $\gamma$ indicates a stronger directional dependence at that point. From the visual inspection of the MHVRs, the difference is noticeable when $\gamma$ exceeds 0.7 , and the difference is significant if $\gamma$ exceeds 1.0. The value of $\gamma$ for each point is denoted in the right-top corner in Figs. 4, 5, 6 and 7. The points along Line-C (running north to south close to the central part of Fig. 9) show large values of $\gamma$ as well as the points within the premises of the Onahama Port Office (the gray hatched block surrounded by thicker lines in Fig. 9). The points that have large $\gamma$, i.e., points in yellow to red, are aligned in a line and concentrated in some parts of the studied area.

\section{Direction of the largest directionally dependent coefficient $\gamma$} In the previous section, the directionally dependent coefficient $\gamma$ was calculated based on the original direction of the microtremor measurement, which is NS and EW components. The value of $\gamma$ can be a useful index to clarify the directional dependence by using these two components, but in this section, we calculate $\gamma$ for different directions by rotating the NS axis from $-45^{\circ}$ to $45^{\circ}$, in order to find the actual orientation that strongly relates to the directional dependence. In Fig. 10, the value of $\gamma$ for each degree from $-45^{\circ}$ to $45^{\circ}$ for points C3, G3 and $\mathrm{H} 3$ is shown. For points C3 and G3, for which $\gamma$ is relatively large, the maximum value of $\gamma$ is close to $0^{\circ}$ and $\gamma$ is greater than 0.7 for the angular range of about $70^{\circ}$, i.e., $-35^{\circ}$ to $40^{\circ}$ and $-35^{\circ}$ to $35^{\circ}$ for C3 and G3, respectively. For point $\mathrm{H} 3, \gamma$ barely reaches above 0.7 over the entire angular range. This means that even if we do not rotate the coordinates when we calculate $\gamma$ and use NS/ $\mathrm{UD}$ and EW/UD, we are likely to detect the directional 

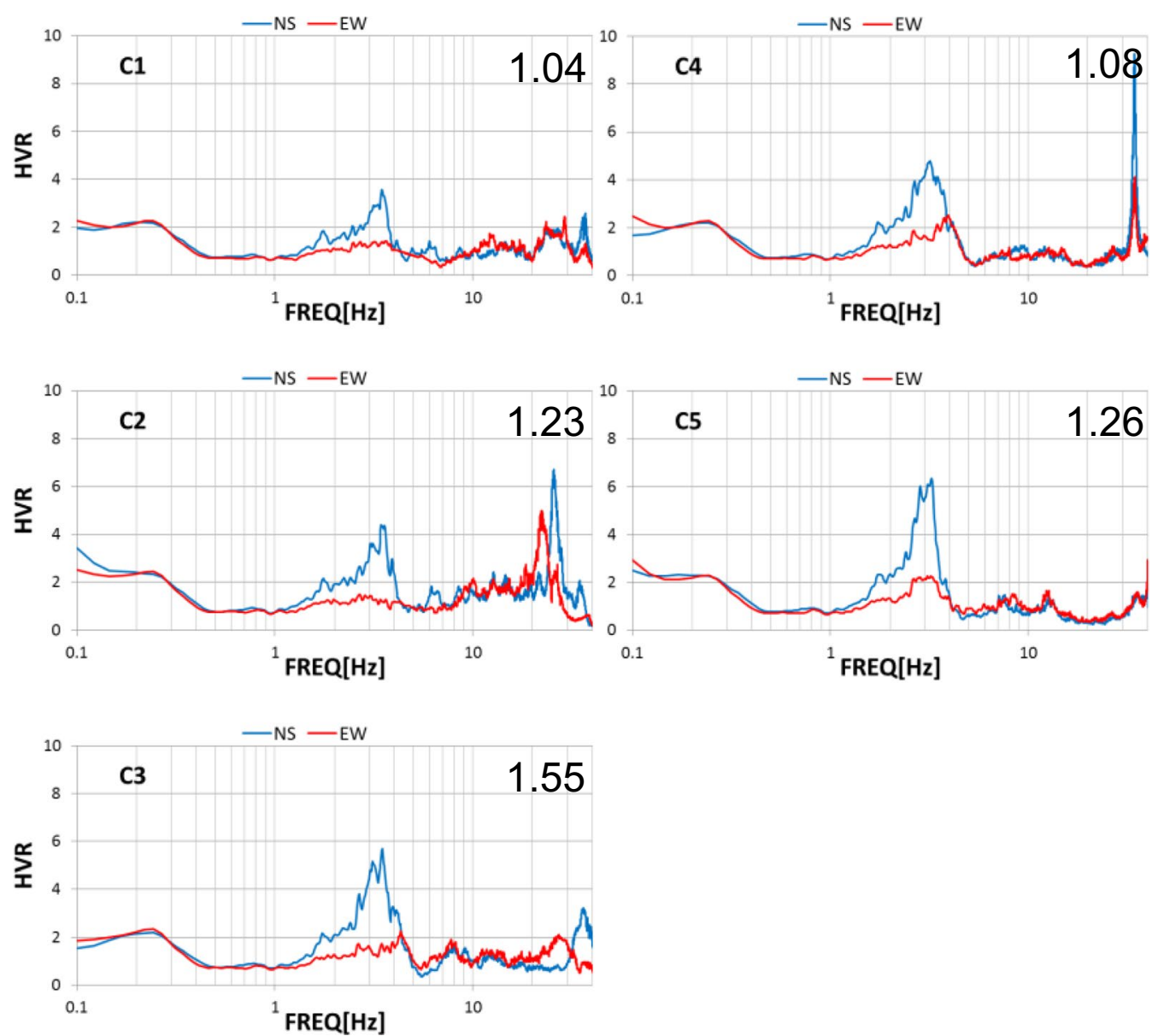

Fig. 7 Comparison of MHVRs along Line-C shown in Fig. 3. The figures from top-left to bottom-right are aligned from west to east on the survey line. Red and blue lines indicate NS/UD and EW/UD. The difference between the two is significant at all points. The value of $\gamma$ for each point is denoted in the right-top corner

dependence from the value of $\gamma$, if MHVRs have strong directional dependence.

Although $\gamma$ can be used to determine the magnitude of the directional dependence, it cannot be used to determine the direction which has the larger amplitude among the two orthogonal components. In Fig. 11, the largest $\gamma$ value after rotating the coordinate and the direction of the axis with the larger and smaller amplitudes are shown by the length of the two orthogonal lines at each point. In this way, we can see the direction in which the difference between the two orthogonal directions is largest and also see the direction with the larger amplitude. As we can see from Fig. 11, the points where the larger axis is oriented in the NS direction are themselves aligned in the NS direction and equivalently for EW direction. The overall effect is a T-shaped distribution. From these results, we infer the possible existence of relatively narrow lateral heterogeneity running along this $\mathrm{T}$ shape. The dotted line denotes the value of 0.7 .

\section{Theoretical MHVRs}

Analytical MHVRs by one-dimensional velocity structure

In this section, we aim to simulate the peak frequency and peak amplitude of the observed MHVRs by theoretical MHVRs obtained from analytical solution based on the diffuse field assumption (Sánchez-Sesma et al. 2008, 2011). The velocity model at the observation points was constructed based on an identified velocity structure by previous study from MASW analysis (Kosaka 2015). Table 1 shows the velocity model used for theoretical calculation, which was simplified to 1 layer above the layer with $V_{\mathrm{s}}=937.1 \mathrm{~m} / \mathrm{s}$, from the results of the previous study. We focus on three points, C3, E2 and F4 as a representative point with directional dependency, without directional dependency whose peak frequency is about $10 \mathrm{~Hz}$ and without directional dependency whose peak frequency is about $12 \mathrm{~Hz}$, respectively, to estimate the depth of the shallow layer at these points. For C3, we 


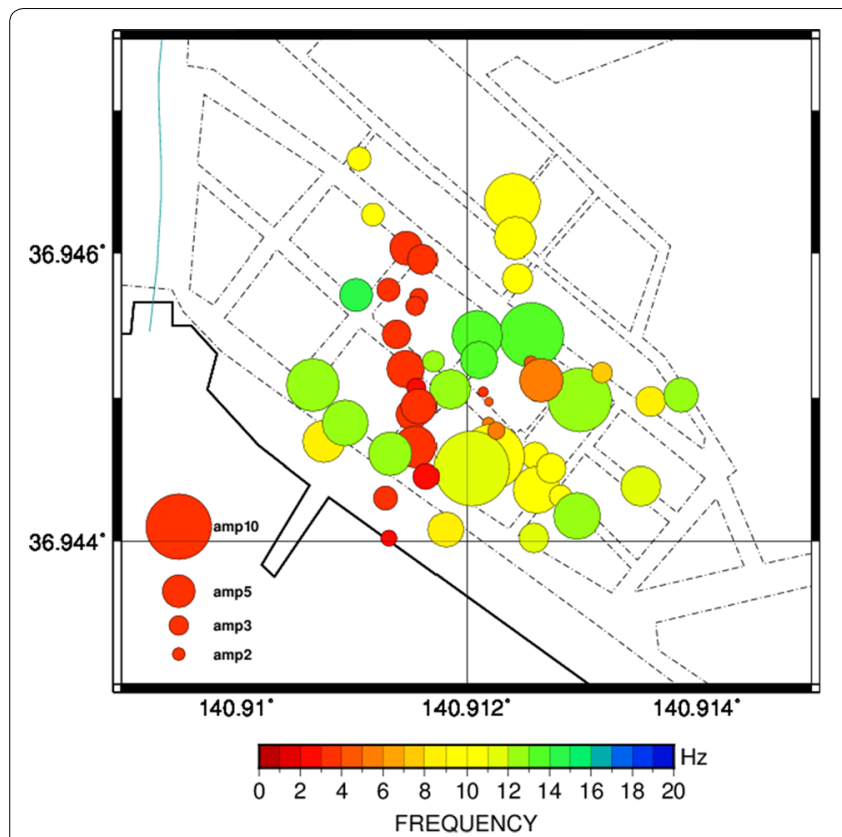

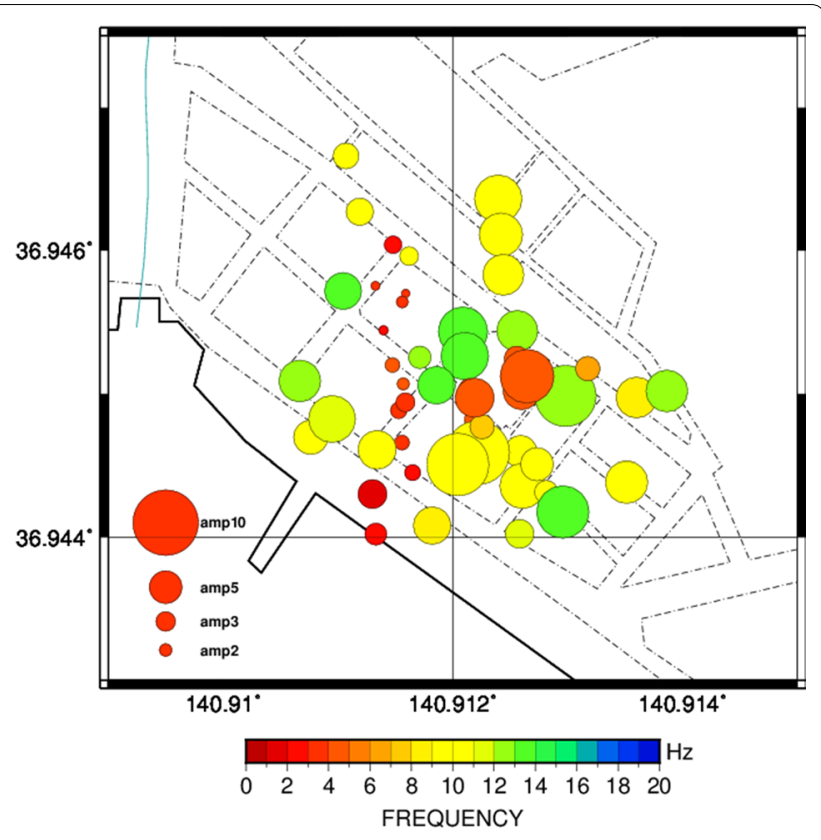

b

Fig. 8 Distribution of the peak frequency and the peak amplitude of $\mathbf{a}$ NS/UD and $\mathbf{b}$ EW/UD of MHVRs for points in Fig. 3. The colors indicate the peak frequency, and the size of the circles indicates the amplitude at the peak frequency

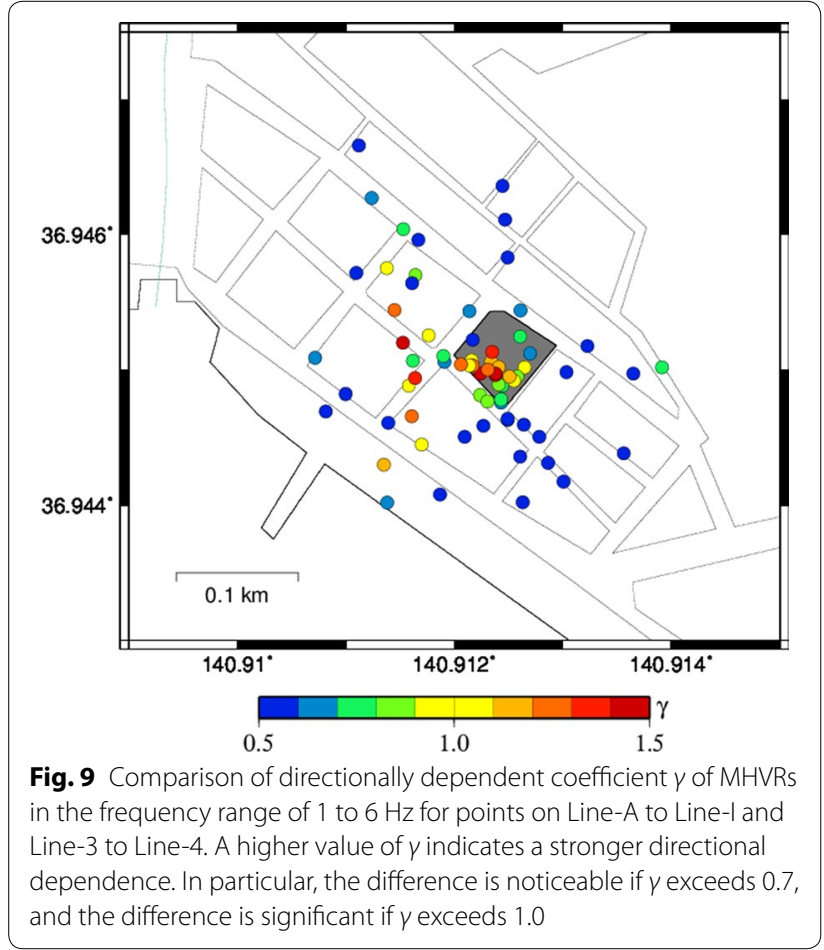

only take into account of the peak frequency of NS/ $\mathrm{UD}$, at about $3.5 \mathrm{~Hz}$, since it can be considered that EW/UD is distorted because of the effect of the strong lateral heterogeneity (Matsushima et al. 2014). In order to fit the peak frequencies at these three points, we determined the depth of Layer 1 to be 15,5 and $4 \mathrm{~m}$ for points C3, E2 and F4, respectively.

Figure 12 shows the comparison between the observed and theoretical MHVRs. The theoretical MHVRs were calculated assuming a 1-D velocity structure at each point. We can see that the observed MHVRs are fairly well reproduced by the theoretical MHVRs considering the simplified velocity structure around the study area. This shows that the depth of the first layer is shallow at $5 \mathrm{~m}$ for point E2 and becomes deep at $15 \mathrm{~m}$ for point C3 and then gets shallow again at $4 \mathrm{~m}$ for point $\mathrm{F} 4$. If we look at Fig. 3, we can see that the depth changes rapidly within a very short distance to make a narrow wedge and at point inside the wedge (C3), the observed MHVR shows a significant directional dependency.

\section{Numerical MHVRs by two-dimensional velocity structure in three-dimensional space}

From the results of analytical MHVRs by 1-D velocity structure, we have assumed that some kind of a wedgeshaped structure exists in the studied area. In order to verify this assumption, we constructed a simple 2-D velocity structure in 3-D space. Figure 13 shows the cross section of the wedge, which is $22 \mathrm{~m}$ wide and $15 \mathrm{~m}$ deep. From the borehole survey results (Kosaka 2015), there is a slow velocity layer deeper than $10 \mathrm{~m}$, so we added a 

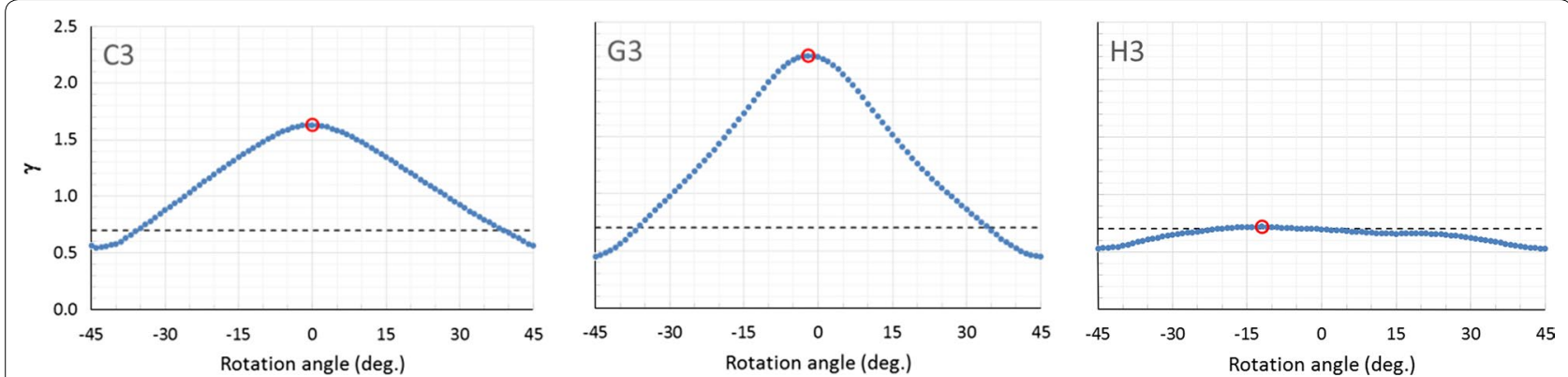

Fig. 10 Transition of $\gamma$ according to the axes rotation angle. For points $C 3$ and $G 3, y$ is largest close to $0^{\circ}$ and remains large over an angular range of about $70^{\circ}$. In contrast, at point $\mathrm{H} 3, y$ is relatively small throughout whole angular range

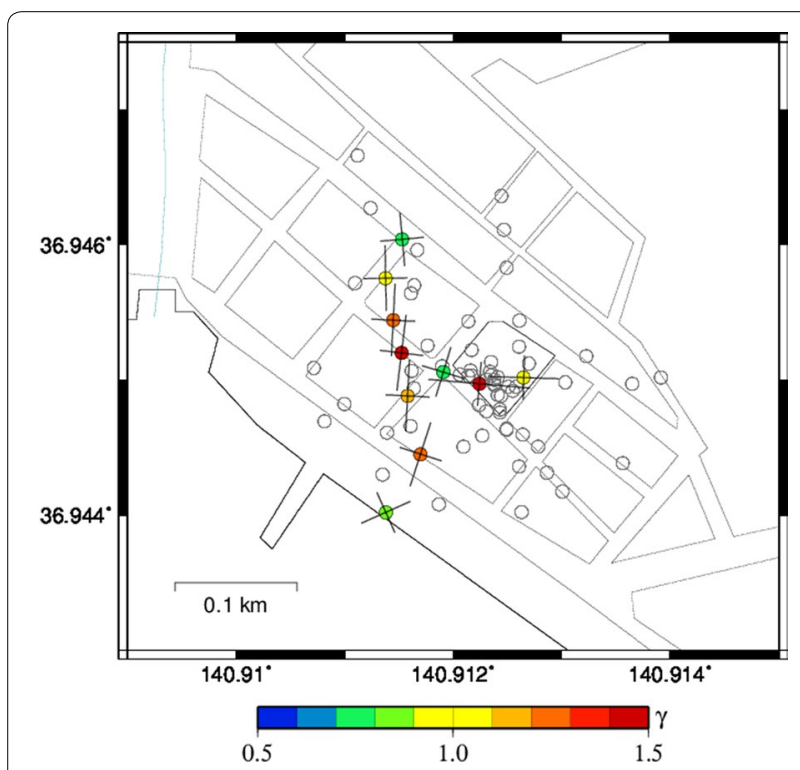

Fig. 11 Largest $\gamma$ value and axes direction of the largest and smallest amplitude for the corresponding direction. Points for which the largest axis is oriented in the NS direction are aligned in the NS direction, and the points where largest axis is oriented in the EW direction are aligned in the EW direction, forming a T-shaped distribution

Table 1 Velocity model used for 1-D analytical calculation

\begin{tabular}{lcll}
\hline & $\boldsymbol{V}_{\mathbf{p}}(\mathbf{m} / \mathbf{s})$ & $\boldsymbol{V}_{\mathbf{s}}(\mathbf{m} / \mathbf{s})$ & Density $\left(\mathbf{k g} / \mathbf{m}^{\mathbf{3}}\right)$ \\
\hline Layer 1 & 816.4 & 203.5 & 1710 \\
Layer 2 & 2411.0 & 937.1 & 2050 \\
\hline
\end{tabular}

layer with $V_{\mathrm{s}}=110.0 \mathrm{~m} / \mathrm{s}$ with a thickness of $5 \mathrm{~m}$ in the deepest part of the wedge. The velocity structure for the 2-D model is listed in Table 2. We use a spectral element method (SEM) (De Martin 2011) called "EFISPEC3D" (http://efispec.free.fr). The conditions for the SEM calculation are listed in Table 3. The mesh is composed by unstructured hexahedra, designed to be accurate with five Gauss-Lobatto-Legendre (GLL) nodes per wavelength at $20 \mathrm{~Hz}$. We use polynomials of order of four to sample the wave field; therefore, each hexahedron contains $5 \times 5 \times 5=125$ GLL nodes. The minimum size of the mesh in the $V_{\mathrm{s}}=110.0 \mathrm{~m} / \mathrm{s}$ layer is $4 \mathrm{~m}$. In order to use the diffuse field assumption to calculate the numerical MHVRs, we need to calculate the imaginary part of the Green's function. We calculate the response at the same point as the source excited by the first derivative of hyperbolic tangent function with a duration of $0.2 \mathrm{~s}$ and deconvolve the response to get the Green's function. Figure 14 shows the MHVRs calculated using SEM at points E2, C3 and F4. For points E2 and F4, there is no significant difference between NS/UD and EW/UD as seen in the observed MHVRs and the peak frequency fits the observed ones fairly well. As for point C3, the numerical MHVRs show good correspondence with the directional dependency seen in the observed MHVRs, although the peak frequency of NS/UD is slightly higher than the observed. If we assume that the wedge is running EW, instead of NS, we can compare the observed MHVRs at point A6 with the numerical MHVRs at point C3, as shown in Fig. 15. The difference between NS/UD and EW/UD is also well reproduced, although the peak frequency is slightly lower than the observed.

\section{Discussions}

From the results shown in Figs. 14 and 15, we can conclude that the numerical MHVRs correspond reasonably well to the observed MHVRs, both inside and outside of the wedge. This suggests that the model and method used to calculate the numerical MHVRs in this study are appropriate. The differences between NS/UD and EW/UD at points $\mathrm{C} 3$ and A6 are significant, and the numerical MHVRs are able to simulate the large difference between the two components. The component with the larger amplitude is parallel to the direction of the wedge, and the component with the smaller 

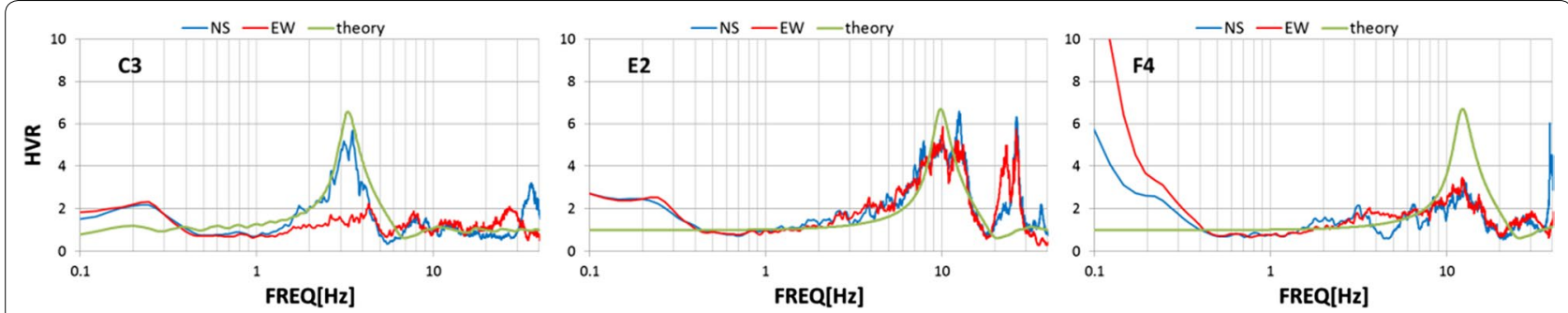

Fig. 12 Comparison of the observed MHVRs (blue and red lines) with the analytical MHVRs (green line) for points C3, E2 and F4. The peak frequency and peak amplitude fit the NS/UD at point C3, both components at point E2 and the peak frequency at point F4

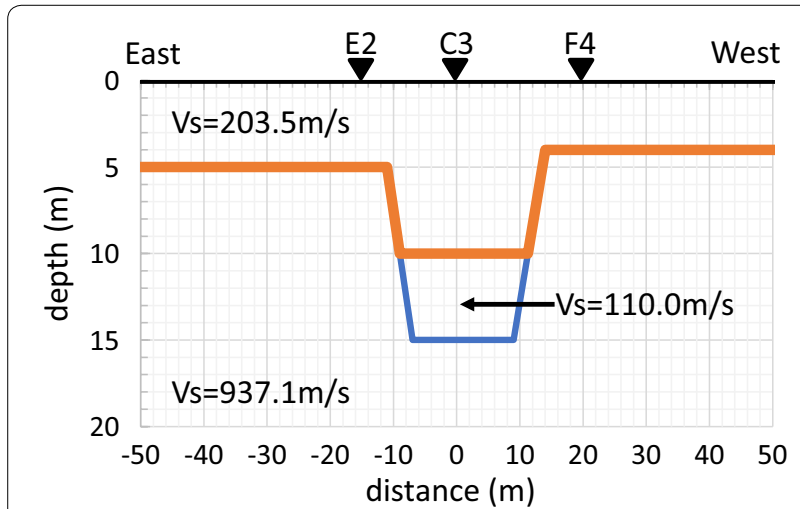

Fig. 13 Cross section of the 2-D wedge used for 3-D SEM calculation. The relative location of points E2, C3 and F4 is shown when we assume that the wedge is in the NS direction

Table 2 Velocity model used for 3-D SEM calculation

\begin{tabular}{lcll}
\hline & $\boldsymbol{V}_{\mathbf{p}}(\mathbf{m} / \mathbf{s})$ & $\boldsymbol{V}_{\mathbf{s}}(\mathbf{m} / \mathbf{s})$ & Density $\left(\mathbf{k g} / \mathbf{m}^{\mathbf{3}}\right)$ \\
\hline Layer 1 & 816.4 & 203.5 & 1710 \\
Layer 2 & 441.3 & 110.0 & 1622 \\
Layer 3 & 2411.0 & 937.1 & 2050 \\
\hline
\end{tabular}

Table 3 Conditions for the 3-D SEM calculation

\begin{tabular}{ll}
\hline Parameter & \\
\hline Model size & Length $\times$ width $\times$ depth \\
& $840 \mathrm{~m} \times 840 \mathrm{~m} \times 840 \mathrm{~m}$ \\
Minimum element size & $4 \mathrm{~m}$ \\
Total elements & 494,429 \\
Order of polynomial's shape function & 4 \\
Maximum frequency & $20 \mathrm{~Hz}$ \\
Minimal number of GLL nodes at $20 \mathrm{~Hz}$ & 5 \\
Time increment & $0.000035 \mathrm{~s}$ \\
Time step & 142,858 \\
Total duration & $5.0 \mathrm{~s}$ \\
\hline
\end{tabular}

amplitude is perpendicular to the direction of the wedge. This shows that the wedge has a large effect on the directional dependence of the MHVRs. In contrast, the peak frequency of the numerical MHVRs was not perfectly fit to the observed MHVRs, so the wedge model needs to be modified. Several trials of numerical calculations showed that the peak frequency is affected not only by the depth of the wedge and the velocity structure within the wedge, but also by the width of the wedge. This implies that fitting the observed MHVRs will require considering the width and depth of the wedge. Although it will be a difficult task to identify the actual wedge structure, it is clear that a relatively narrow lateral heterogeneity may exist in the shallow subsurface beneath these points, parallel to the direction of the larger amplitude.

\section{Conclusions}

In this study, we observed MHVRs at a strong motion observation site of the Port and Harbor Research Institute in Onahama, Japan, which is one of the sites that were selected for detailed study in the European project "PRENOLIN." We found that directional dependence exists in some parts of the area surrounding the site. The directional dependence is significant and has a dominant frequency at around $5 \mathrm{~Hz}$.

We conducted microtremor observations to detect the distribution of the directionally dependent MHVRs to find the cause of the directionality. We defined "directionally dependent coefficient $\gamma$ " with which to classify sites according to their directional dependence. The magnitude of the directional dependence corresponding to the value of $\gamma$ is implied by the difference between the two orthogonal components and can be derived from the MHVRs. Points of which $\gamma$ is larger correspond to points with stronger directional dependence that can be seen from the MHVRs in Figs. 4, 5, 6 and 7. The MHVRs of points on Line-C show large $\gamma$ values and have higher amplitude for NS/UD compared to EW/UD. In addition, the MHVRs on Line-4 also show large $\gamma$ values, again 

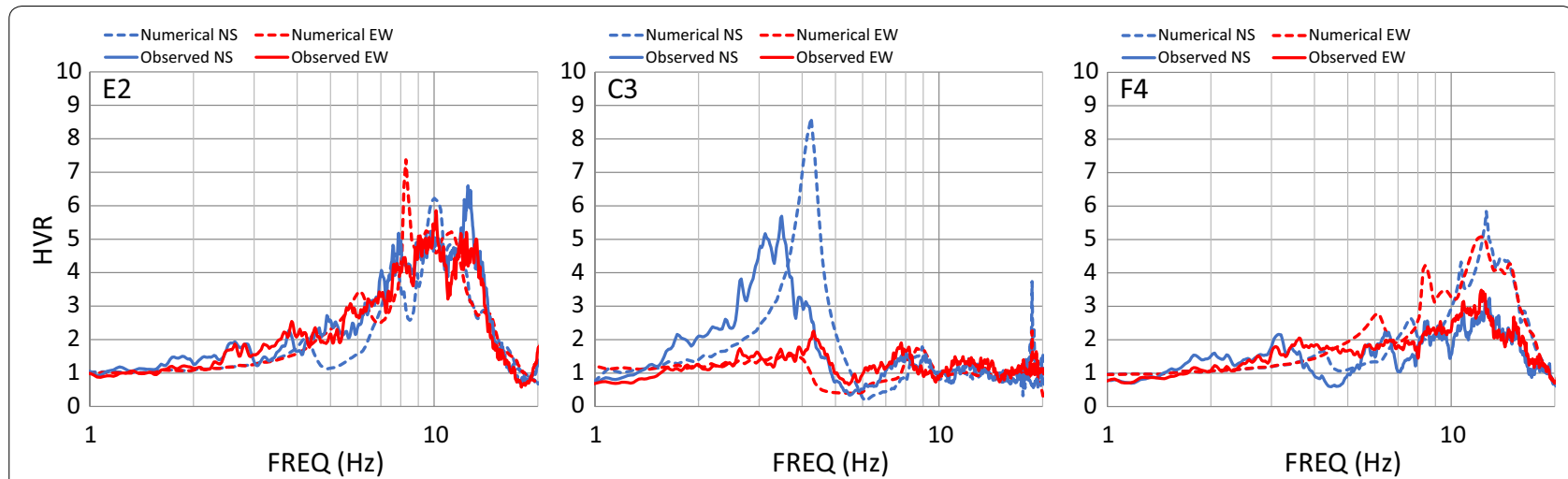

Fig. 14 Comparison of the observed MHVRs (solid blue and red lines) with the numerical MHVRs (broken blue and red lines) for points E2, C3 and F4. There is effectively no difference between NS/UD and EW/UD for points E2 and E4, and the numerical MHVRs fit the observed ones. As for point C3, the numerical MHVRs show the same directional dependence as the observed

corresponding to the directional dependence that can be seen in Fig. 4, but EW/UD has larger amplitude compared to NS/UD. Points for which the largest axis is oriented in the NS direction are aligned in the NS direction, and points for which the largest axis is oriented in the EW direction are aligned in the EW direction, forming a T-shaped distribution. To simulate the observed MHVRs, we first calculated the analytical MHVRs assuming a 1-D velocity structure. As a result, we showed the possible existence of a narrow wedge from the MHVRs. Next, we calculated the numerical MHVRs assuming a 2-D wedge shape in 3-D space to simulate the directionally dependent MHVRs. As a result, we calculated the numerical

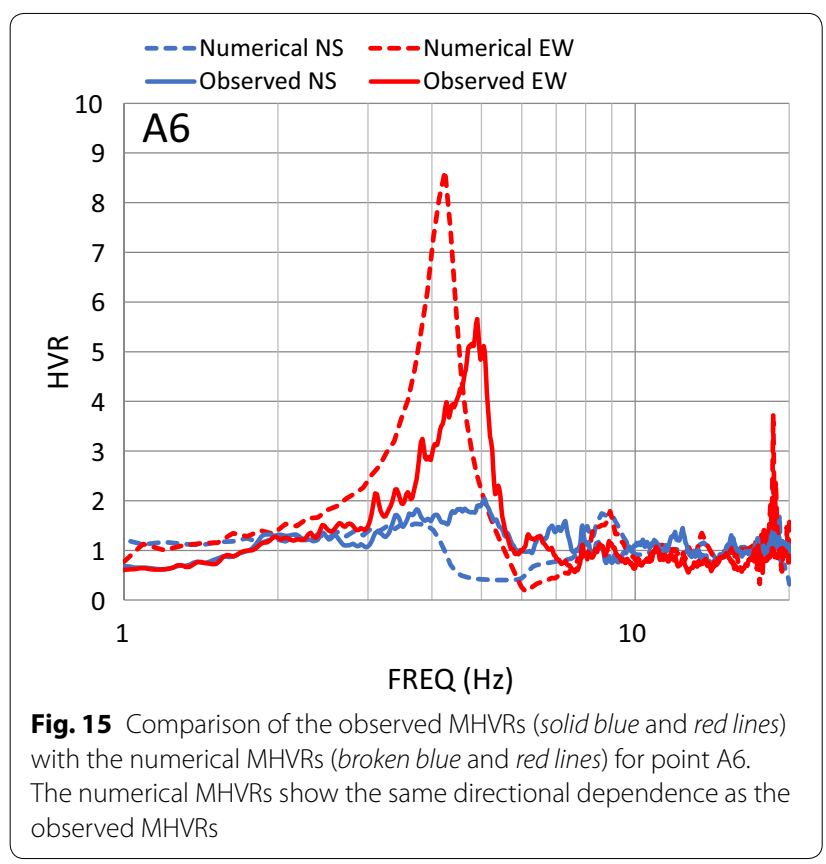

MHVRs that were in fairly good correspondence with the observed MHVRs, although the velocity model needs to be modified to fit the observed MHVRs perfectly. From these results, we conclude that the directional dependence of MHVRs could be a useful tool for detecting the lateral heterogeneity of the subsurface structure.

\section{Abbreviations}

GLL: Gauss-Lobatto-Legendre; MASW: multi-channel analysis of surface waves; MHVR: microtremor horizontal-to-vertical spectral ratio; MLIT: Ministry of Land, Infrastructure, Transport and Tourism; NS: north-south; EW: east-west; UD: up-down; SEM: spectral element method; 1-D: one-dimensional; 2-D: two-dimensional; 3-D: three-dimensional.

\section{Authors' contributions}

Microtremor observations were performed by SM, HKo and HKa. Data analysis was conducted mainly by HKo. Theoretical calculations were performed by SM. SM wrote the draft manuscript through discussions with HKo and HKa. SM, HKo and HKa contributed to edit and revise the manuscript and approved the final submitted manuscript. All authors read and approved the final manuscript.

\section{Author details \\ ${ }^{1}$ Disaster Prevention Research Institute, Kyoto University, Gokasho, Uji, Kyoto 611-0011, Japan. ${ }^{2}$ Toda Institute of Construction Technology, 315 Kaname, Tsukuba, Ibaraki 300-2622, Japan.}

\section{Acknowledgements}

The authors greatly appreciate the kind understanding and cooperation by the Onahama Port Office of Ministry of Land, Infrastructure, Transportation and Tourism. This study was performed in conjunction with the "PRENOLIN Project: a benchmark on numerical simulation of 1-D non-linear site effect" which is a part of two larger projects SINAPS@, funded by the ANR (French National Research Agency) and SIGMA, funded by a consortium of nuclear operators (EDF, CEA, AREVA, ENL). Part of this study uses some of the results of the Bachelor Thesis of Toga Kobayashi. The microtremor observations were conducted with the help of many students of the Kawase-Matsushima laboratory of DPRI, Kyoto University. The analytical calculations were conducted using a source code developed by Prof. Sánchez-Sesma of the National Autonomous University of Mexico (UNAM). Dr. De Martin of the French Geological Survey (BRGM) helped to construct the 2-D velocity structure models for SEM calculation. This study was partially supported by JSPS KAKENHI Grant Numbers JP25420577 and JP16K06573, as well as by internal funds of the Disaster Prevention Research Institute, Kyoto University. 


\section{Competing interests}

The authors declare that they have no competing interests.

\section{Publisher's Note}

Springer Nature remains neutral with regard to jurisdictional claims in published maps and institutional affiliations.

Received: 28 February 2017 Accepted: 6 July 2017

Published online: 13 July 2017

\section{References}

Aki K (1957) Space and time spectra of stationary stochastic waves, with special reference to microtremors. Bull Earthq Res Inst Univ Tokyo 35(3):415-456

De Martin F (2011) Verification of a spectral-element method code for the southern California earthquake center $\mathrm{LOH} .3$ viscoelastic case. Bull Seismol Soc Am 101(6):2855-2865. doi:10.1785/0120100305

Kosaka H (2015) The spatial variation of shallow subsurface velocity structure derived from microtremor observation and MASW method and its effect to ground motion. Master's Thesis of Graduate School of Engineering, Kyoto University

Matsushima S, Hirokawa T, De Martin F, Kawase H, Sánchez-Sesma FJ (2014) The effect of lateral heterogeneity on horizontal-to-vertical spectral ratio of microtremors inferred from observation and synthetics. Bull Seismol Soc Am 104(1):381-393. doi:10.1785/0120120321
Nozu A, Wakai A (2011) Annual report on strong motion earthquake records in Japanese ports (2010). Technical Note of the Port and Airport Research Institute, No. 1243 (in Japanese with English abstract)

Régnier J, Bonilla LF, Bard P-Y, Kawase H, Bertrand E, Hollender F, Marot M, Sicilia D and Nozu A (2015) PRENOLIN Project: a benchmark on numerical simulation of 1D non-linear site effects. 2-Results of the validation phase. In: 6th International conference on earthquake geotechnical engineering 1-4 November 2015 Christchurch, New Zealand, 103

Sánchez-Sesma FJ, Pérez-Ruiz JA, Luzón F, Campillo M, Rodríguez-Castellanos A (2008) Diffuse fields in dynamic elasticity. Wave Motion 45:641-654. doi:10.1016/j.wavemoti.2007.07.005

Sánchez-Sesma FJ, Rodríguez M, Iturrarán-Viveros U, Luzón F, Campillo M, Margerin L, García-Jerez A, Suarez M, Santoyo MA, Rodríguez-Castellanos A (2011) A theory for microtremor H/V spectral ratio: application for a layered medium. Geophys J Int Exp Lett 186(1):221-225. doi:10.1111/j.1365-246X.2011.05064.x

\section{Submit your manuscript to a SpringerOpen ${ }^{\odot}$ journal and benefit from:}

- Convenient online submission

- Rigorous peer review

- Open access: articles freely available online

- High visibility within the field

- Retaining the copyright to your article

Submit your next manuscript at $\boldsymbol{\nabla}$ springeropen.com 